\title{
Perceptual rivalry with vibrotactile stimuli
}

\author{
Farzaneh Darki $^{1} \mathbb{D}$. James Rankin ${ }^{1}$
}

Accepted: 8 February 2021

(C) Crown 2021

\begin{abstract}
In perceptual rivalry, ambiguous sensory information leads to dynamic changes in the perceptual interpretation of fixed stimuli. This phenomenon occurs when participants receive sensory stimuli that support two or more distinct interpretations; this results in spontaneous alternations between possible perceptual interpretations. Perceptual rivalry has been widely studied across different sensory modalities including vision, audition, and to a limited extent, in the tactile domain. Common features of perceptual rivalry across various ambiguous visual and auditory paradigms characterize the randomness of switching times and their dependence on input strength manipulations (Levelt's propositions). It is still unclear whether the general characteristics of perceptual rivalry are preserved with tactile stimuli. This study aims to introduce a simple tactile stimulus capable of generating perceptual rivalry and explores whether general features of perceptual rivalry from other modalities extend to the tactile domain. Our results confirm that Levelt's proposition II extends to tactile bistability, and that the stochastic characteristics of irregular perceptual alternations agree with non-tactile modalities. An analysis of correlations between subsequent perceptual phases reveals a significant positive correlation at lag 1 (as found in visual bistability), and a negative correlation for lag 2 (in contrast with visual bistability).
\end{abstract}

Keywords Vibrotactile $\cdot$ Perceptual rivalry $\cdot$ Levelt's propositions $\cdot$ Scaling property $\cdot$ Correlation analysis

\section{Introduction}

Multistable perception occurs when sensory information is ambiguous and consistent with two or more perceptual states. In this phenomenon, perception alternates intermittently between two (bistable) or more (multistable) interpretations of the fixed stimulus (Sterzer et al., 2009). Examples of multistable perception are well known in vision such as motion direction with plaids (ambiguous barber poles) (Hupé \& Rubin, 2003; Wuerger et al., 1996), apparent motion (Meso et al., 2016; Ramachandran \& Anstis, 1983), the Necker cube (Toppino, 2003), and binocular rivalry (Blake, 1989), and span across other sensory modalities including audition (Pressnitzer \& Hupé, 2006) and olfaction (Zhou \& Chen, 2009). All of these multistable phenomena share common features, such as exclusivity of perceptual interpretations, randomness, inevitability of alternations, independence of perceptual

Farzaneh Darki

fd303@exeter.ac.uk

1 Department of Mathematics, College of Engineering, Mathematics and Physical Sciences, University of Exeter, Exeter, UK phases (Lathrop, 1966; Fox \& Herrmann, 1967), and Levelt's propositions (Levelt, 1965; Leopold \& Logothetis, 1999). These similar characteristics give rise to the conclusion that perceptual ambiguity must have a common neural basis and is likely resolved at a higher level of cognition that is not specific to individual sensory modalities (Pressnitzer \& Hupé, 2006; Logothetis et al., 1996a; Wolfe, 1996). However, a more recent study suggests that perceptual switching arises from a distributed system of similar but independent processes (Denham et al., 2018). As far as we know, these theories have been limited to the visual or auditory domains, and no previous research has investigated the possible outcomes with tactile stimuli.

Many of the early studies of tactile perception were focused on investigating the existence of tactual equivalents of apparent motion illusions. These kind of illusory experiments were generally extensions of known illusions from vision (Lederman \& Klatzky, 2009). In visual experiments, two spatially separated lights flash on and off in turn and can produce the illusion of movement (also known as phi phenomenon or beta movement). A tactile variant of smooth apparent motion was first produced with stimulation of two vibrotactile bursts of $150 \mathrm{~Hz}$ presented sequentially on the skin (Boring, 1942; Sherrick \& Rogers, 1966). Observers typically report that the series of discrete 
tactile vibrations feel like vibratory stimulation moving across the skin (Burtt, 1917; Kirman, 1974; Lederman \& Jones, 2011; Burtt, 1917). Various stimulus parameters such as the inter-stimulus onset interval (ISOI) and stimulus duration (from 25 to $400 \mathrm{~ms}$ ) have been studied to find the optimal ranges for the induction of smooth apparent movement (Sherrick \& Rogers, 1966). Apparent motion can also happen with a bilateral stimulus delivered to each arm, however, the movement is less robust than the movement reported when stimulation is delivered to a unilateral limb such as a single thigh or a single arm (Sherrick, 1968). These observations from illusory experiments provide insight into mechanisms leading to perceptual alternations (bistability) with tactile stimuli.

Tactile pulses separated in location and time can induce a sensation of movement. For more complicated patterns, where different directions of movement are consistent with pulses, perception can be bistable. The first example of an apparent motion stimulus that leads to perception of motion in different directions is the "apparent motion quartet" stimulus realized in both the visual and tactile domains (Carter et al., 2008). In different studies, pairs of vibrotactile stimuli were attached to the tip of a participant's index finger (Carter et al., 2008), at locations on both index fingers (Conrad et al., 2012), to the thumbs and index fingers (Haladjian et al., 2020), or to both forearms (Liaci et al., 2016). The position of each consecutive stimulus pair alternated between the opposing diagonal corners of an imaginary rectangle which leads to switches between the perception of motion traveling either horizontally or vertically (Fig. 1a). The proximity of stimulus pairs is the strongest contributor to the direction in which apparent motion is perceived, with motion more likely to be experienced between closer stimulus pairs than more distant ones (Gengerelli, 1948).

There are numerous stimulus examples for perceptual ambiguity that show similar properties across sensory modalities and across different paradigms within the same sensory modality. Levelt's propositions have been broadly used to describe perceptual rivalry in the visual (MorenoBote et al., 2010; Brascamp et al., 2015) and auditory (Rankin et al., 2015) domains. For example, the generalization of Levelt's proposition II states that increasing the difference between percept strengths increases the mean perceptual dominance of the stronger percepts (Levelt, 1965). Despite mean dominance times varying widely in multistable experiment, across different observers and stimulus contrasts (Zhou et al., 2004; Brascamp et al., 2005), the distribution of perceptual phases maintains a constant shape (gamma-like distribution) (Blake et al., 1980; Cao et al., 2016; Denham et al., 2018). Previous studies in vision suggested that the durations of successive perceptual phases are statistically independent (insignificant correlation) (Logothetis et al., 1996b; Pressnitzer \& Hupé, 2006), however, recent studies of binocular rivalry (van Ee, 2009; Cao et al., 2020) revealed positive correlations for perceptual phases that were one phase apart (between different percepts). Auditory streaming experiments also show positive correlations for durations separated by one phase and negative correlations for durations that are two phases apart (between same percepts) (Barniv \& Nelken, 2015). To the best of our knowledge, no previous research has investigated these stochastic properties of perceptual rivalry in tactile modality.

Levelt's propositions and other common characteristics in multistable perception have yet to be explored with tactile stimuli. We hypothesize that Levelt's propositions will extend to tactile rivalry appropriately chosen stimulus parameter manipulations. Here we present a new ambiguous tactile stimulus paradigm, similar to beta motion from vision, presented at only two locations on the skin but still capable of producing tactile bistability during minuteslong trials. Whilst beta motion in vision results from a fixed intensity (contrast) moving dot, the stimulus presented here involves alternating changes in intensity (vibration amplitude) at the locations on each index finger. Two different perceptual interpretations can arise that compete for dominance over time. Because of the simplicity of this tactile stimulus, it is well suited to investigate the mechanisms underlying tactile rivalry. We varied intensity difference $(\Delta I)$ asymmetrically during different trials as a control parameter to assess its effect on perceptual durations. We aim to characterize the stochasticity of perceptual alternations by looking at properties of the distribution of switching times. Our results show that general characteristics of bistable perception such as Levelt's proposition II (LVII) and stochastic characteristics like a scaling property hold for the tactile modality. This provides a new avenue to expand studies of the general mechanisms underlying neural competition across multiple sensory modalities.

\section{Methods}

\section{Power analysis}

The factors tested in our experiments had not been tested in any earlier studies, so we carried out a preliminary experiment with six participants. For each participant, we had six repetitions at each intensity difference level $(\Delta I=$ $2,4,6 d B)$, resulting in 36 samples for each condition. For calculations of partial $\eta^{2}$, we used the effectsize package in $\mathrm{R}$ to perform a two-way repeated measures ANOVA. 
The reported partial $\eta^{2}$ values in Table 1 correspond to the following Cohen's measurements of effect size $f($ percept $)=.58, f(\Delta I)=.31, f(\Delta I:$ percept $)=.77$.

We used the minimum effect of $\eta^{2}=.09$ in the preliminary experiment to estimate the sample size. The correlation between means of SIM and AM perceptual durations is -.43 . In order to detect an effect of partial $\eta^{2}=.09$ with $85 \%$ power in a two-way within-subjects ANOVA (5 groups, $\alpha=.05$, non-sphericity correction $=$ 1), G*Power (Faul et al., n.d.) suggests we would need 14 participants.

\section{Participants}

Fifteen volunteers ( 8 male, mean age $29.07 \pm 7.43$ SD) were recruited from the University of Exeter. Each gave written informed consent and received minor monetary compensation for participating in a 1-h session. Participants were naive to the purpose of the study and did not selfdeclare any neurological or sensory disorders. Procedures were in compliance with guidelines for research with human subjects and approved by University of Exeter Research Ethics Committee.

\section{Experiment design and procedure}

Participants sat in an acoustically isolated booth and attended to vibrotactile stimulators attached to their right and left index fingers. We used miniature vibrotactile electromagnetic solenoid-type stimulators $(18 \mathrm{~mm}$ diameter, Dancer Design tactors, www.dancerdesign.co.uk) driven by a tactile amplifier (Dancer Design Tactamp) to deliver stimuli. Vibrotactile stimuli consisted of sequences of $400 \mathrm{~ms}$ high (H) and low (L) intensity $200 \mathrm{~Hz}$ vibratory pulses, each followed by a $400 \mathrm{~ms}$ silent interval (H-L-H$\mathrm{L}$ for the right hand and L-H-L-H for the left hand, "-" indicates the silent gap) (Fig. 1b). The intensity of the $\mathrm{L}$ stimulus was $\Delta I$ below the intensity of the $\mathrm{H}$ stimulus on a logarithmic scale $(d B)$. We chose the full-amplitude based on a value where differences in amplitude were noticeable in unambiguous perception. The voltages applied to the tactors for full-amplitude $200 \mathrm{~Hz}$ sinusoidal vibration was
3.38 V. To mask any unwanted low-intensity sound emitted by the vibrotactile stimuli, participants listened to pink noise played through noise-isolating headphones at a comfortable listening level. During a trial participants' perception of the stimulus changed over time and they reported their current perceptual interpretation with key presses (sampled at $100 \mathrm{~Hz}$ ) on a keyboard (Fig. 1c). Participants perceived the stimulus as either one simultaneous pattern of vibration on both hands (SIM), or patterns of vibration that jumped from one hand to the other hand, giving a sensation of apparent movement (AM) (Fig. 1d). Subjects were instructed to report their percepts passively and not try to hold one perceptual interpretation over another.

During experimental sessions, 3-min trials were repeated three times in blocks of five trials for a range of intensity differences $(\Delta I=.5,1,2,4,6 d B)$ in random order. For a given participant, this resulted in a total of 15 trials $(45 \mathrm{~min}$ total trial time). The interval between trials was a minimum of $20 \mathrm{~s}$. We used a $5 \times 5$ Latin square design with nine randomized and unique grids so that the order of conditions for each participant in a block of trials was counterbalanced within/across participants and repetitions.

\section{Data analysis}

In the first stage of our analysis, every trial with average percept durations larger than $150 \mathrm{~s}$ (above dashed line in Fig. 2a) or smaller than $4 \mathrm{~s}$ (equivalent to H-L-H-L-H-) were excluded from data set before further analysis (91 trials were excluded from the total of 225 trials). For each of 15 subjects, we take the average percept duration across all repetitions. So, each subject contributes a mean SIM and a mean AM duration averaged across all durations for the three repetitions at each condition. Other measures such as proportion of time with SIM or AM percept, and the frequency of switches are computed in a similar fashion. To see the effect of discarding trials with mean SIM and mean AM durations below $4 \mathrm{~s}$ and beyond $150 \mathrm{~s}$ compare Fig. 2 with Fig. S1 in supplementary material (no qualitative change).

In order to establish whether the parameter $\Delta I$ had a significant effect on the measures described above,

Table 1 Two-way repeated measure ANOVA of mean duration of both percept types (AM, SIM) with respect to intensity difference ( $\Delta I)$ and percept type for the preliminary experiment

\begin{tabular}{llllll}
\hline Source & $d f_{\text {num }}$ & $d f_{\text {den }}$ & $F$ & $p$ & $\eta_{p}{ }^{2}$ \\
\hline (Intercept) & 1 & 35 & 210.13 & $<.001$ & $<.001$ \\
percept & 1 & 35 & 41.50 & $<.001$ & $.25 \pm .08$ \\
$\Delta I$ & 2 & 70 & 15.74 & $<.001$ & $.09 \pm .06$ \\
$\Delta I$ :percept & 2 & 70 & 67.92 & $.37 \pm .07$ \\
\hline
\end{tabular}

Analysis shows a significant effect of percept and also $\Delta I$ on the mean durations 
a

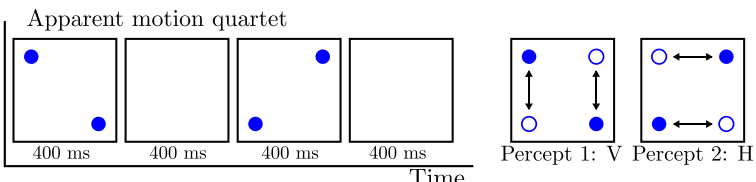

b Simplified vibrotactile stimuli

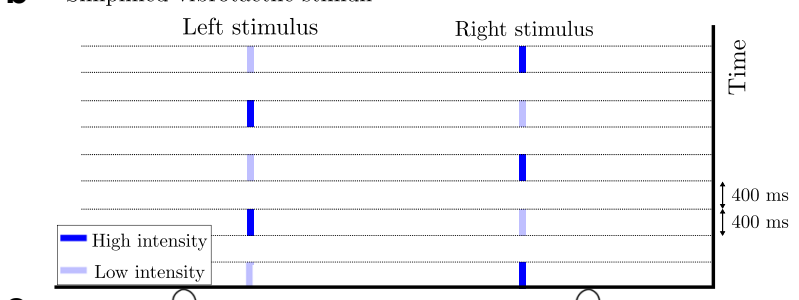

C

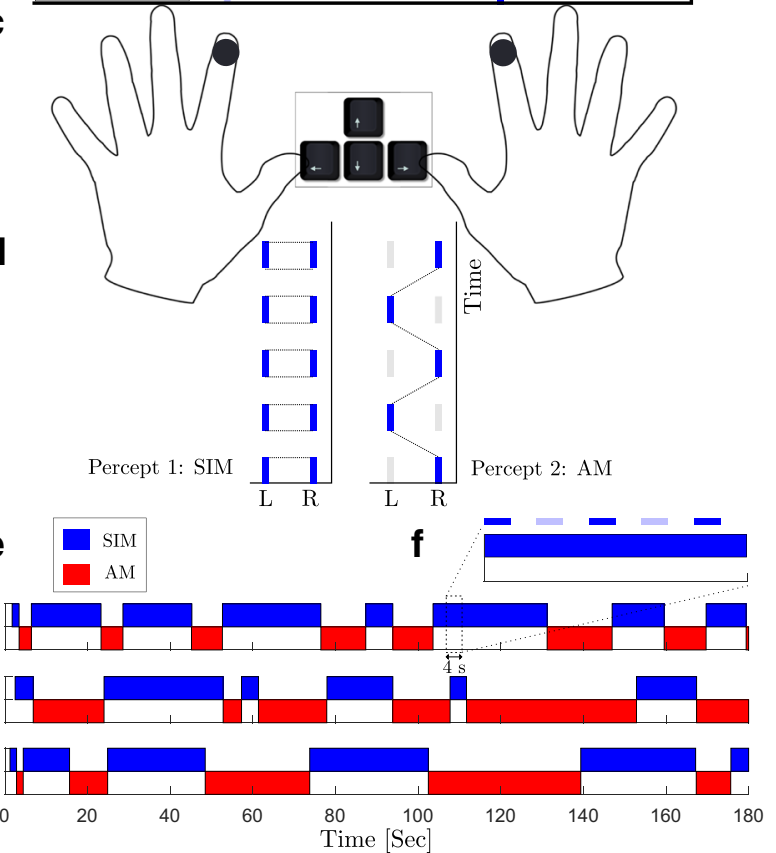

Fig. 1 a Apparent motion quartet. The stimulus consists of a cycle of four stimulus frames. Two dots appear at the diagonal corners of an invisible square during stimulus interval, then disappear during the inter-stimulus interval (ISI), reappear at the opposing corners and disappear during the ISI. The stimulus is perceptually ambiguous and can yield either horizontal or vertical motion percepts (indicated by the black arrows). For the tactile motion quartet, four stimulus locations on e.g., a subject's fingers are used with similar timing intervals. b Vibrotactile stimuli. Sequences of high (dark blue) and low-intensity (light blue) of 200-kHz vibrations are delivered to the right and the left index finger. c Experimental setup. Vibrotactile stimuli are delivered to subject's index fingers. During a trial subject's perception of the stimuli changes, they report it by holding appropriate keys on the keyboard using their thumbs. d Percept types. When the patterns are played with equal intensity, they can be perceived as one simultaneous vibration (SIM). With a fixed intensity difference $(\Delta I>0 d B)$ between the high- and low-intensity tactile pulses, perception switches back and forth between two percepts: SIM (perceived as a fixed intensity on each hand, even though the intensity is changing) and AM (perceived as pulses of vibrations jumping from one hand to the other hand). We associate the left arrow key with SIM and the right arrow key with AM. e Perceptual phases. Perceptual interpretations of the stimuli for three different subjects during 3-min trials at $\Delta I=2 d B$. f Relative scale of the stimuli. A 4-s zoomed panel which shows the relation between the stimulus and the perceptual phases we used repeated measure ANOVA with Bonferroni corrections. As we had discarded some of the trials (due to unacceptably large or small means), we resampled data from mean percept durations at each experimental condition to substitute discarded trials and balance the number of observations across experimental conditions. ANOVA tables and post hoc analyses are reported in full in Tables S1-S7 (supplementary material). A significance level of .05 is used throughout this paper. In ANOVA tables the Greenhouse-Geisser (GG) corrected $p$ values are reported if a Mauchly sphericity (MS) test reached significance. In post hoc analyses quoted $p$ values are Bonferroni corrected to account for multiple comparisons. Standard measures of effect size (generalized eta-squared) are quoted for statistically significant results. All statistical analyses were carried out in the statistical package R.

The distributions of normalized percept durations of both types shown in Fig. 3 were compared with gamma and lognormal distributions using a one-way Kolmogorov-Smirnov $(\mathrm{KS})$ test. The null hypothesis is that the test data are drawn from the standard distribution and a significant result $(p<$ .05 ) indicates that the test data are not drawn from the comparison distribution.

In order to check for a scaling property, we first need to compute central moments. The distribution shape of samples t may be quantified in terms of the mean $\mu_{1} \equiv$ $\mathbb{E}[t]$ and the central moments $\mu_{2} \equiv \mathbb{E}\left[\left(t-\mu_{1}\right)^{2}\right]$, $\mu_{3} \equiv \mathbb{E}\left[\left(t-\mu_{1}\right)^{3}\right]$, etc., or, equivalently, in terms of normalized moments, such as the coefficient of variation $c_{v}=\mu_{2}{ }^{1 / 2} / \mu_{1}$ and the skewness $\gamma_{1}=\mu_{3} / \mu_{2}{ }^{3 / 2}$. A scaling property obtains if central moments are proportional to corresponding powers of the mean or, equivalently, if normalized moments are constant as follows:

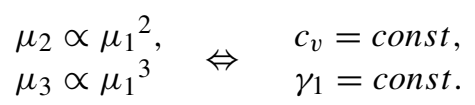

The normalization of percept durations was used in order to combine data across participants and different experimental conditions (Rankin et al., 2015), whilst accounting for some of the subject variability in experiments (supplementary material Figure S2). For correlation analysis, durations were normalized to the average value of each percept type separately within each trial and subject, in order to avoid spurious correlation due to inter-subject and inter-trial differences in switching behavior. Here we used the Pearson correlation that measures the strength and direction of the linear relationship between two variables. Pearson's linear correlation coefficient $C$ orr is defined as:

$\operatorname{Corr}(x, y)=\frac{\Sigma\left(x-\mu_{x}\right)\left(y-\mu_{y}\right)}{\sqrt{\Sigma\left(x-\mu_{x}\right)^{2} \Sigma\left(y-\mu_{y}\right)^{2}}}$,

where $\mu_{x}$ and $\mu_{x}$ are the means of variables $x$ and $y$. 
a

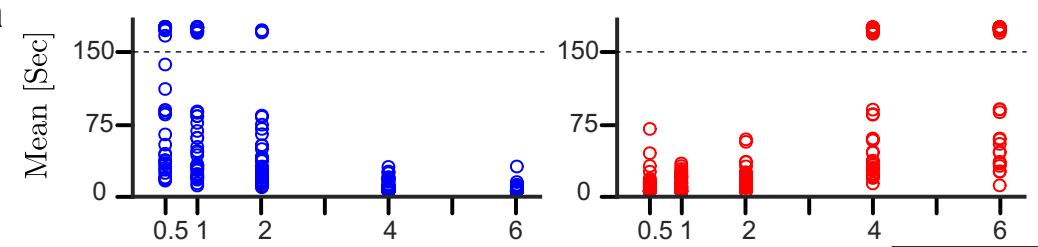

b
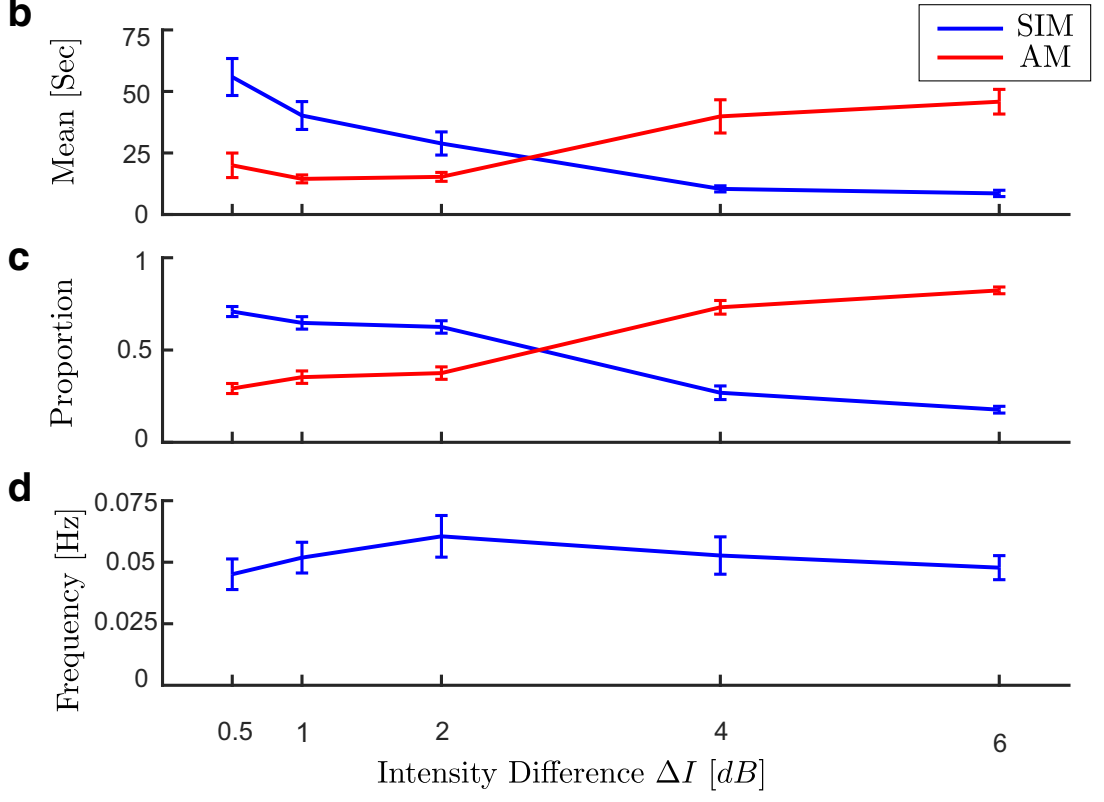

Fig. 2 a The distribution of dominance durations at each trial with different experimental conditions (45 observations per experimental condition). All the samples above the dashed line (larger than $150 \mathrm{~s}$ ), were excluded from the data set before further analysis (for similar analysis without excluding data see Figure S1 in supplementary materials). It is assumed that durations longer than $150 \mathrm{~s}$ are from trials where perception did not alternate. $\mathbf{b}$ Mean dominance duration, $\mathbf{c}$ proportion of dominance for each percept type, $\mathbf{d}$ alternation rate, as a function of intensity difference $(\Delta I)$

To ensure consistency of our method, we also computed the correlations with a second method (Fig. 4e-h) based on correlations in single trials without normalization. As correlations may not be reliable as computed for single trials in isolation, we treated each trial as a sample of the correlation and tested whether the mean of the distribution was significantly above or below zero.

\section{Results}

The experiment with antiphase sequences of high and low intensity tactile stimuli on the right and the left index finger showed irregular perceptual switches between two interpretations for a range of the parameter $\Delta I$. The average percept duration across all participants and trials for both percept types was $17.9 s \pm 22.7 s$, which spans on average over 35 periods of the stimuli (Fig. 1e and f). The average percep duration across all participants for both percept types at $\Delta I=2 d B$ was $15.8 s \pm 19.4 s$. The fraction of time spent in SIM was .52. For some participants at small or large
$\Delta I$ values perception may not alternate, these trials were excluded from dataset.

\section{Levelt's proposition II}

To establish whether Levelt's proposition II extends to the tactile domain, we chose intensity difference $(\Delta I)$ as a control parameter and examined the temporal dynamics of perceptual alternations. The distribution of mean perceptual dominance time in each trial across both percept types and $\Delta I$ conditions is plotted in Fig. 2a. Mean dominance duration, proportion of time spent in each percept type and alternation rate are plotted against intensity difference $(\Delta I)$, respectively in Fig. 2b-d. Increasing intensity difference $(\Delta I)$ (intensity of the $\mathrm{H}$ stimulus is fixed, intensity of the $\mathrm{L}$ stimulus is decreased), causes the mean dominance of SIM percept to decrease and AM percept to increase (Fig. 2b). A similar pattern is shown for proportion of perceptual dominance (Fig. 2c). The alternation rate reaches a maximum at equidominance $\Delta I=2 d B$ (the point where each percept approximately dominates half the time), 

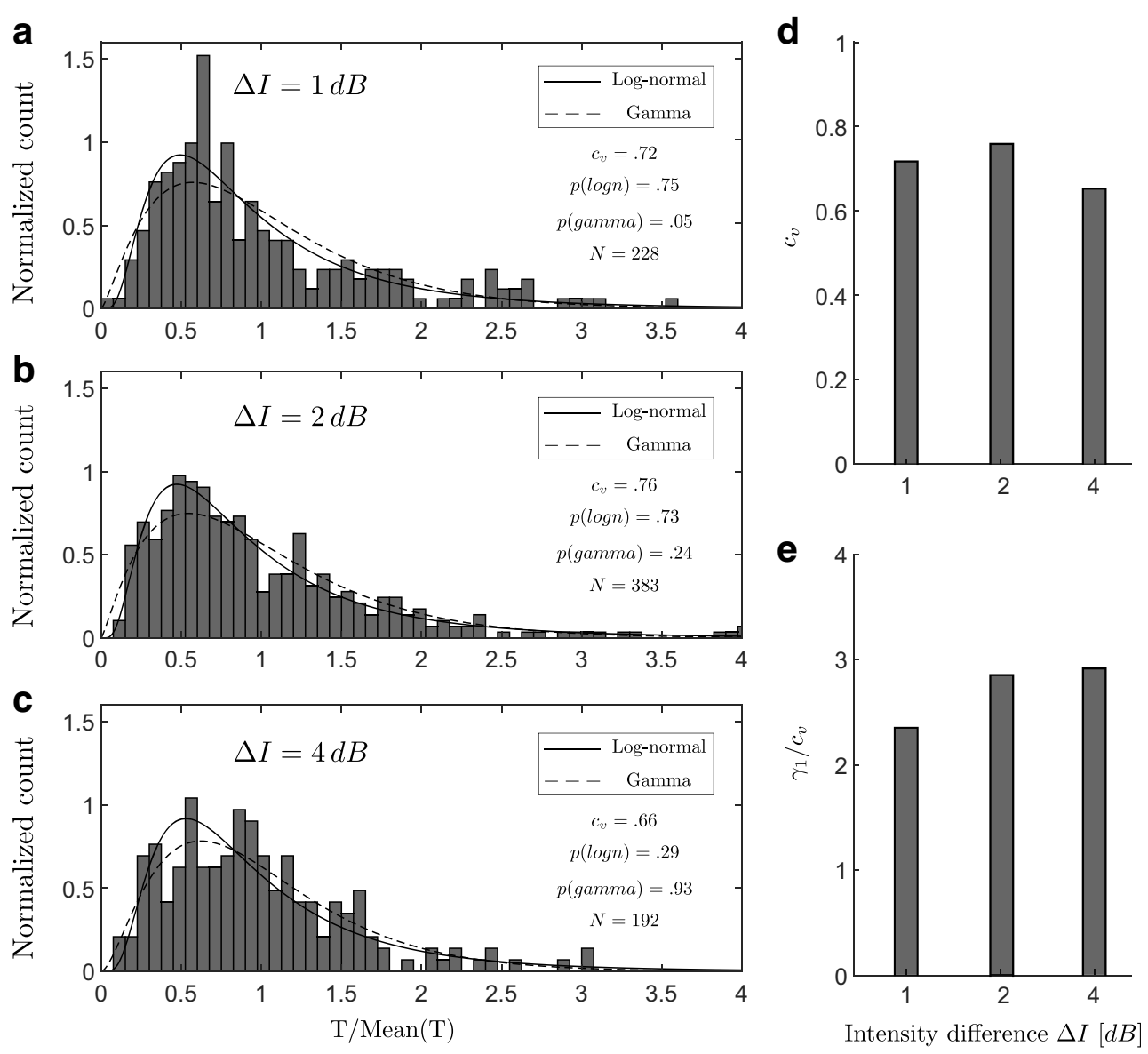

Fig. 3 Histograms of normalized perceptual phases for experimental conditions close to equidominance, a $\Delta I=1 d B$, b $\Delta I=2 d B$, and c $\Delta I=4 d B$ combined across participants and percept type after normalization by the mean. Solid and dashed curves show the estimated log-normal and gamma distribution, respectively. $\mathbf{d}$ Coefficient of variation $\left(c_{v}\right)$ and e skewness divided by coefficient of variation $\left(\gamma_{1} / c_{v}\right)$ for experimental conditions $\Delta I=1,2,4 d B$

and decreases symmetrically below and above this point (Fig. 2d). Of the conditions tested $\Delta I=2 d B$ is closest to equidominance; from panels $\mathrm{B}$ and $\mathrm{C}$ in Fig. 2 it appears $\Delta I=3 d B$, if tested, would be closer still to equidominance and have a higher alternation rate. Results from our experiments demonstrate that Levelt's proposition II holds in tactile domain.

A two-way repeated measures ANOVA of mean duration of both percept types (SIM, AM) was performed with Percept type (SIM, AM) and intensity difference $\Delta I$ as within-subjects factors. The analysis reported in Table 2 shows a significant interaction for $\Delta I$ :percept, $F(4,164)=$ 50.01, $p<.001$. As for the individual factor, percept does not reach significance $F(1,41)=3.34, p=.07$, however for the individual factor $\Delta I$ reaches significance, $F(4,164)=3.93, p=.004$. A one-way repeated measures ANOVA of mean duration of SIM percept was performed with $\Delta I$ as the within-subjects factor. The analysis reported in Table 3 shows a significant effect of $\Delta I$ on mean dominance SIM $F(4,164)=25.04, p<.001($ Similar

Table 2 Two-way repeated measure ANOVA of mean duration of both percept types (AM, SIM) with respect to intensity difference $(\Delta I)$ and percept type

\begin{tabular}{|c|c|c|c|c|c|c|}
\hline Source & $d f_{\text {num }}$ & $d f_{d e n}$ & $F$ & $p$ & ges & $p[G G]$ \\
\hline Percept & 1 & 41 & 3.34 & .07 & & \\
\hline$\Delta I$ & 4 & 164 & 3.93 & .004 & .01 & .01 \\
\hline$\Delta I:$ Percept & 4 & 164 & 50.01 & $<.001$ & .32 & $<.001$ \\
\hline
\end{tabular}

Analysis shows a significant effect of $\Delta I$ and also $\Delta I$ :percept on the mean durations 
Table 3 One-way repeated measure ANOVA of mean duration of SIM perception with respect to intensity difference $(\Delta I)$

\begin{tabular}{lllllll}
\hline Source & $d f_{\text {num }}$ & $d f_{\text {den }}$ & $F$ & $p$ & ges & $p[G G]$ \\
\hline$\Delta I$ & 4 & 164 & 25.04 & $<.001$ & .03 & $<.001$
\end{tabular}

Analysis shows a significant effect of the intensity difference on the mean durations

results for AM percept in Table 5). Pairwise comparisons with Bonferroni-corrected significance levels revealed that each individual condition has significant differences for all non-adjacent conditions and for the pair $\Delta I=[2,4] d B$ (Table 4, Similar results for AM percept in Table 6). Taken together, these results demonstrate a significant main effect of varying $\Delta I$ on mean dominance duration (Tables 5 and 6) and proportion of dominance of each percept (Tables 2 and 3 in Supplementary Material).

\section{Statistics of dominance durations and scaling property}

Perceptual phases for bistable stimuli have been shown to be fit well by gamma or log-normal distributions. However, experiments with large numbers of participants found the log-normal distribution to be a better fit across visual and auditory bistable stimuli (Denham et al., 2018). The distributions of normalized perceptual phases for experimental conditions close to equidominance are shown in Fig. 3a-c. In the temporal analysis of perceptual durations for bistable stimuli, the perceptual phases are normalized by the mean for each percept type (see Fig. S2 in supplementary material for normalization method). The coefficient of variation $\left(c_{v}\right)$, which is the ratio of the standard deviation to the mean, is used as a measure of variability in the perceptual phases. The result of oneway KS tests shows that, except for the gamma standard distributions at $\Delta I=1 d B($ p $($ gamma $)<.05)$, all the other histograms are compatible with the comparison distributions.

To assess how well tactile rivalry conforms to the scaling property reported in (Cao et al., 2014; Cao et al., 2016), we compared observations from three intermediate

Table 4 Pairwise $t$ test, with Bonferroni corrected $p$ values, on the mean durations of SIM perception with respect to intensity difference $(\Delta I)$

\begin{tabular}{lllll}
\hline & $\Delta I=.5$ & $\Delta I=1$ & $\Delta I=2$ & $\Delta I=4$ \\
\hline$\Delta I=1$ & .28 & - & - & - \\
$\Delta I=2$ & .001 & .45 & - & - \\
$\Delta I=4$ & $<.001$ & $<.001$ & .004 & - \\
$\Delta I=6$ & $<.001$ & $<.001$ & .001 & 1.0 \\
\hline
\end{tabular}

Table 5 One-way repeated measure ANOVA of mean duration of AM perception with respect to intensity difference $(\Delta I)$

\begin{tabular}{lllllll}
\hline Source & $d f_{\text {num }}$ & $d f_{\text {den }}$ & $F$ & $p$ & ges & $p[G G]$ \\
\hline$\Delta I$ & 4 & 164 & 27.26 & $<.001$ & .34 & $<.001$ \\
\hline
\end{tabular}

Analysis shows a significant effect of the intensity difference on the mean durations

experimental conditions $(\Delta I=1,2,4 d B)$. The extreme conditions were discarded from further analysis, as they had fewer perceptual phases leading to inaccurate computation of second and third moments. A scaling property obtains if normalized moments, such as the coefficient of variation $c_{v}$ and ratio of skewness and coefficient of variation $\gamma_{1} / c_{v}$ are constant. Figure $3 \mathrm{~d}$ and e illustrates the results in terms of the coefficient of variation and skewness across different experimental conditions. The coefficient of variation remained consistently near $c_{v}=.6$ (Fig. 3d) and ratio of skewness and coefficient of variation $\gamma_{1} / c_{v}$ remained roughly constant. Figure $3 \mathrm{e}$. In other words, a scaling property was maintained over intermediate experimental conditions.

\section{Analysis of correlation}

Figure 4a-d plots the normalized duration of each perceptual phase against the duration of the next, for the two possible transitions (lag1: $\mathrm{SIM} \rightarrow \mathrm{AM}$ and $\mathrm{AM} \rightarrow \mathrm{SIM}$ ). Importantly, durations were normalized to the average value of each percept type separately within each trial and subject, in order to avoid spurious correlations due to intersubject and inter-trial differences in switching behavior (see Methods). For consecutive phase durations (lag 1), the correlation was small but significantly larger than zero (Fig. 4a and b). For the phase durations that were one phase apart (lag 2: $\mathrm{SIM} \rightarrow \mathrm{SIM}$ and $\mathrm{AM} \rightarrow \mathrm{AM}$ ), the correlation was significantly smaller than zero (Fig. $4 \mathrm{c}$ and d).

We also calculated correlation coefficients separately for each switch type in each individual trial. Figure $4 \mathrm{e}-\mathrm{h}$ shows histograms of the single trial correlations between subsequent phases (lag 1) and between phase durations that are one phase apart (lag 2). Using a t-statistic, we found

Table 6 Pairwise $t$ test, with Bonferroni corrected $p$ values, on the mean durations of $\mathbf{A M}$ perception with respect to intensity difference $(\Delta I)$

\begin{tabular}{lllll}
\hline & $\Delta I=.5$ & $\Delta I=1$ & $\Delta I=2$ & $\Delta I=4$ \\
\hline$\Delta I=1$ & 1.0 & - & - & - \\
$\Delta I=2$ & 1.0 & 1.0 & - & - \\
$\Delta I=4$ & $<.001$ & $<.001$ & $<.001$ & - \\
$\Delta I=6$ & $<.001$ & $<.001$ & $<.001$ & .017 \\
\hline
\end{tabular}



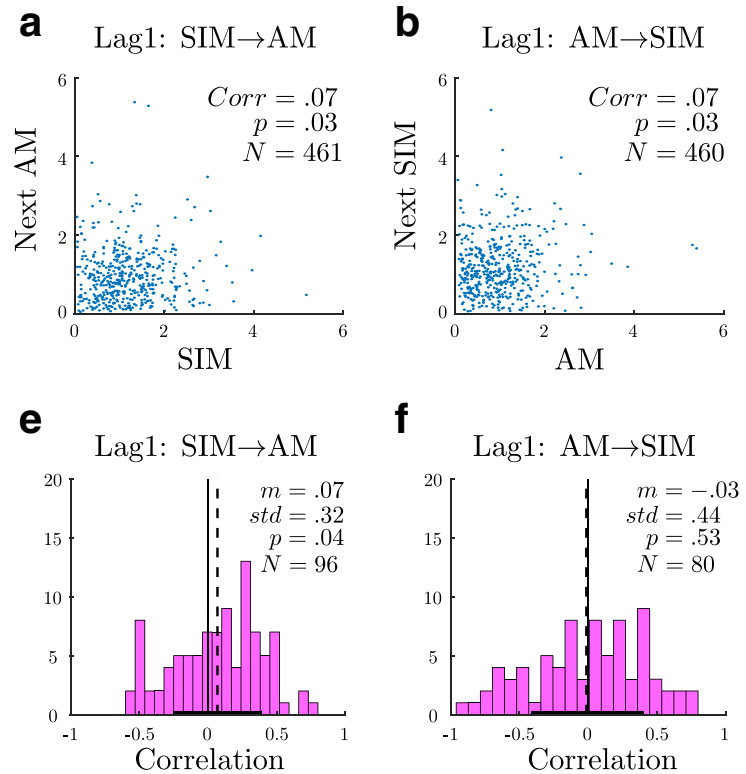

Fig. 4 a-d Scatter plots of normalized durations. The correlation coefficient (corr) between perceptual phases for each scatter is indicated in each panel with the corresponding $p$ value and number of pairs ( $p$ and $N$, respectively). e-h Histograms of correlation coefficients between perceptual phases in single trials. The mean $(m)$ and standard deviation $(s t d)$ are indicated in each panel, followed by the

a significant deviation of the histogram towards positive correlation values for lag 1; SIM $\rightarrow$ AM transition (Fig. 4e), and negative correlation values for lag2; SIM $\rightarrow$ SIM transition (Fig. 4h). Single trial correlations for lag1; $\mathrm{AM} \rightarrow \mathrm{SIM}$ and lag $2 ; \mathrm{AM} \rightarrow \mathrm{AM}$ were not significantly different from zero.

\section{Discussion}

\section{Summary}

Earlier studies with tactile stimuli have identified bistability in somatosensation; these have typically investigated the spatial proximity of stimulation sites as a control parameter. Future studies are needed to characterize the full range of spatial and temporal stimulation parameters capable of inducing bistable tactile apparent motion. Here we introduced a simple tactile stimulus which can evoke bistability through stimulating only two sites (in comparison with four sites in previous studies). We investigated the effect of varying intensity difference $(\Delta I)$ asymmetrically on perceptual durations. Our results show that Levelt's proposition II (LVII) and other characteristics of sensory bistability that generalize in vision and auditory sensation extend to the tactile modality.
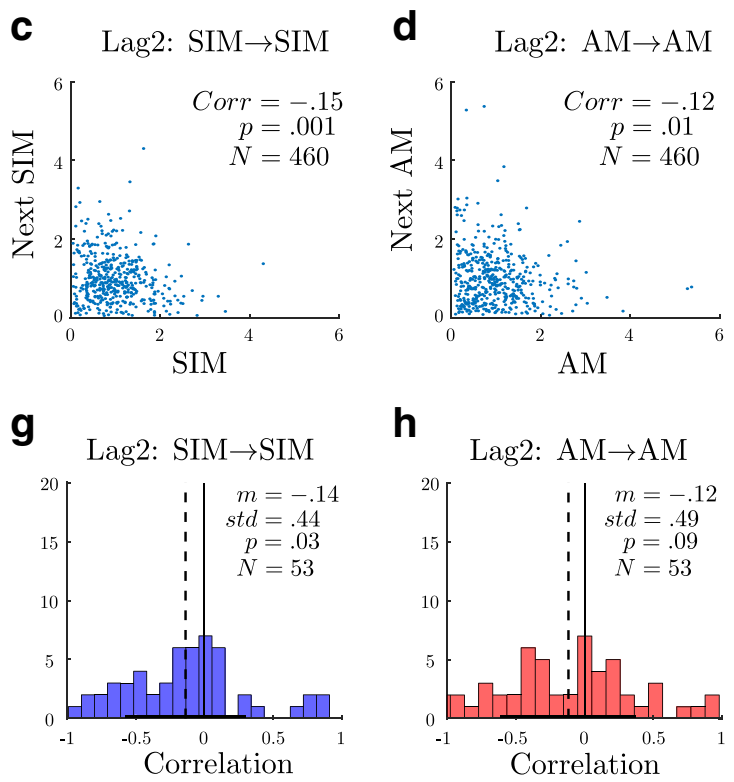

t-statistic of the fixed effect, its significance $(p)$ and the number of trials for which it was possible to calculate the correlation $(N)$. The vertical solid lines mark zero correlation, and the vertical dashed lines mark the mean of each distribution. The transition types are marked above each histogram a\&e AM following SIM. b\&f SIM following AM. c\&g SIM. d\&h AM

\section{Novelty of the introduced stimuli and experiment design}

In this study, we introduced a new ambiguous tactile stimulus, consisting of anti-phase sequences of high- and low-intensity vibration pulses on the right and left index fingertips. While in similar work on tactile bistability stimuli were presented on at least four sites on the skin (Carter et al., 2008; Liaci et al., 2016), our stimulus is simpler and was only presented at two sites. Our approach probes how motion perception is affected by feature (intensity) differences other than just location. This could be extended to look at other features, e.g., vibration frequency, temporal cues, presentation rate, etc. Another important difference is in the way participants reported their perception and also in the analyses of perceptual responses. In one study, participants were asked to attend to the stimuli and report their perception at the end of short representations of tactile stimuli (Liaci et al., 2016). In another study, participants reported their perception continuously during long representations of the stimuli, however; the analysis considered temporal evolution of the average responses pooled across all participants (Carter et al., 2008), which does not account for the dynamics of individual perceptual durations and inter-subject variability. In neither study is the intensity difference varied as a control 
parameter, but the results of our experiment show that it significantly affects the higher-level interpretation of the stimulus. Here, our analysis involves the temporal durations of perceptual phases and investigates the effect of varying the intensity difference $(\Delta I)$ asymmetrically on perceptual durations.

\section{Similar properties of perceptual competition across different modalities}

Perception of the tactile stimuli showed the following similarities to other sensory modalities. First, visual, auditory and tactile stimuli can induce the perception of apparent motion which can be ambiguous in a certain range of stimulus parameters, for example here with a synchronous percept (SIM). Second, Levelt's proposition generalizes to other visual modalities including ambiguous motion (Moreno-Bote et al., 2010) and to auditory bistability (Rankin et al., 2015). Here our analysis showed Levelt's proposition II extends to include tactile bistability. Third, in the experiments of visual and auditory bistability, the distribution of perceptual phases maintains a characteristic, gamma-like shape (Blake et al., 1980). Denham et al. showed these distributions to be specifically log-normal in experiments with a large number of participants (Denham et al., 2018). Even though mean perceptual phases vary widely between participants and stimulus properties, the variance and skewness of perceptual phases keep a characteristic proportion to the mean (Cao et al., 2014; Cao et al., 2016). Our analysis shows that the scaling property holds in the tactile domain, similar to multistability in other modalities.

Despite these similarities, differences between the other sensory modalities and tactile perception were observed as well. Experiments with an auditory stimulus which utilized sequences of anti-phase high and low tones in each ear showed that the percept can be like a single tone oscillating from ear to ear, which is a form of apparent motion. However, there were other percepts like sensation of either the high tone in the left ear and the low tone in the right ear or vice versa, and the two percepts switching back and forth (Deutsch, 1974). While we used a similar stimulus paradigm, the possible perceptual interpretations that we observed in the tactile domain were different. It remains to be determined whether perceptual interpretations equivalent to those reported in audition can be evoked by different ranges of spectrotemporal features in tactile stimuli. On the other hand, we found that perceptual phases in the tactile modality were generally more stable than in the other bistable perception like visual (Conrad et al., 2012) or auditory modality (Rankin et al., 2015) (mean dominance durations were longer in the tactile experiment). Previous studies in vision (van Ee, 2009; Cao et al., 2020) and auditory streaming (Barniv \& Nelken, 2015) suggested that the durations of successive perceptual phases are positively correlated, however, for lag 2 they have been shown to be negatively correlated in auditory streaming (albeit their result did not prove to be statistically significant) (Barniv \& Nelken, 2015) and to be positively correlated in binocular rivalry (Cao et al., 2020). Our analysis with tactile stimuli shows a significant positive correlation for lag 1 (from one percept type to the other), and a negative correlation for lag 2 (between similar percept types). Existing studies of auditory perception have established that first durations are typically longer than subsequent durations (Pressnitzer \& Hupé, 2006). However, we found no such trend in our data on vibrotactile bistability (see Figure S3 and Table 8 in supplementary material). The effects of attentional focus on dominance durations have been widely reported with other bistable modalities (Toppino, 2003; Meng \& Tong, 2004; Van Ee et al., 2006; Pressnitzer \& Hupé, 2006) but this remains to be explored for tactile bistability.

\section{Locus of tactile rivalry and modeling}

To the best of our knowledge, there are no models for tactile rivalry. Computational models of perceptual ambiguity have helped significantly with our understanding of other types of bistability. In order to develop a computational model of tactile rivalry, one must consider how inputs from the left and right hands project to primary somatosensory cortex (S1) and how features like amplitude, frequency and timing are encoded there. It is known that information from each half of the body-surface is projected to the opposite side of the brain (Maldjian et al., 1999). Ipsilateral stimuli do not travel directly to the neurons in the primary somatosensory cortex (S1) (Eshel et al., 2010). However, an extensive range of research from invasive studies in monkeys (Clarey et al., 1996), to behavioral or neuroimaging studies of humans (Hlushchuk \& Hari, 2006) showed that somatosensory processing in the left side of the brain can be modulated by the right side and vice versa. For instance, during unilateral touch, contralateral activation can be observed as well as ipsilateral deactivation in S1 (Hlushchuk \& Hari, 2006; Kastrup et al., 2008).

In contrast, intracranial recording (Noachtar et al., 1997), functional magnetic resonance imaging (fMRI) (Hämäläinen et al., 2002; Nihashi et al., 2005) and magnetoencephalography (MEG) (Korvenoja et al., 1995; Schnitzler et al., 1995) in humans, demonstrated that unilateral tactile stimulation activates bilateral $\mathrm{S} 1$ response. Moreover, single-cell recording in monkeys showed that some neurons in S1 display bilateral hand receptive fields (Iwamura et al., 2001). These results suggest that interhemispheric connections may not be purely inhibitory for somatosensation and they are likely to have excitatory 
effects as well (Eshel et al., 2010). We might use modeling to check whether purely inhibitory interactions can account for observed behavioral responses, or whether other types of interhemispheric interplay might explain the results instead.

The experiments presented here used tactile pulses at $200 \mathrm{~Hz}$ lasting $400 \mathrm{~ms}$. The neural encoding of location and of spectro-temporal properties of tactile stimuli is relatively well documented. There exist quite a lot of discussions in the literature about the cortical representation of flutter frequencies (less than $80 \mathrm{~Hz}$ ) (Talbot et al., 1968; Romo \& Salinas, 2003), however, it is under debate whether the somatosensory cortices process high frequencies. There is some evidence to suggest that the temporal patterning of the neural activities in primary and secondary somatosensory cortex does not contain information about the frequency of the stimuli in the vibration range (greater than $80 \mathrm{~Hz}$ ) (Ferrington \& Rowe, 1980). The perception of flutter and high frequency vibration may be processed through distinct processing streams (Tommerdahl et al., 2005). Auditory cortex undoubtedly plays a main role in the spectro-temporal representation of acoustic stimuli. There are some hypotheses that the putative neural populations are independently stimulated by tactile and auditory sensory modalities (Yau et al., 2009). The cutaneous vibration frequency can be distinguished at a significantly lower resolution (with Weber fractions around .2 for tactile versus .003 for auditory), and over a narrower range than auditory tone pitch (up to $1 \mathrm{kHz}$ for tactile versus $20 \mathrm{kHz}$ for auditory stimuli) (Saal et al., 2016). The resolution of perception is likely hierarchical as supported by modeling work in visual (Wilson, 2003a) and auditory bistability (Rankin et al., 2015).

There is a need for a tactile rivalry model that accounts for both well-established results on the timing of dominance intervals and is also compatible with the physiological evidence and structure of somatosensation. General models of rivalry usually incorporate a slow process together with reciprocal inhibition to produce perceptual alternations. Perceptual bistability results from a competition between units representing neural populations associated with different percepts (e.g. units driven by inputs from the left and right eyes in binocular rivalry) (Wilson, 2003b; Rankin et al., 2015; Cao et al., 2020). A possible model of tactile rivalry could be based on competition between neural populations in primary somatosensory cortex associated with the right and the left hands. For the development of a model, an important aspect of tactile rivalry is that information is integrated across locations and over time to form the SIM and AM percepts. Dynamical analysis of models with different architecture and connectivity can help us to find a model that induces observed percept types with the specific characteristics and intrinsic dynamics. Our result show that Levelt's proposition II (LVII) holds in the tactile modality. This provides a new avenue to test and expand the computational and neurobiological models for bistability currently dominated by vision science. The correlation structure and statistical distribution properties can be used as an important constraint on models of tactile rivalry, and could reveal mechanistic differences with other modalities.

\section{Conclusions}

We presented a new, simple form of tactile rivalry and explored generalizations with respect to perceptual rivalry in other sensory modalities. First, the results of our study show that Levelt's proposition II which describes the relation between stimulus features and mean perceptual dominance extends to tactile bistability. Second, the stochastic characteristics of irregular perceptual alternations were shown to follow similar distribution shapes across different experimental conditions and with different mean perceptual dominance (known as a scaling property). Third, we found negative correlations for durations that were one phase apart (lag 2) which is opposite to the effect found in vision (Cao et al., 2020). In auditory bistability no significant correlation was found, though it trended in the same direction as the tactile modality results (Barniv $\&$ Nelken, 2015). The paradigm introduced here and the methods of analysis provide a basis to further explore how similar processes generate changes in perception across different senses. This opens up a new avenue for a range of experiments to explore the role of e.g., adaptation and crossinhibition in somatosensation, voluntary control (attention), perceptual memory and the integration of tactile cues with the other senses. This approach will allow for insights gained in previous work from a substantial literature on auditory and visual perception to deepen our understanding of tactile perception.

Abbreviations SIM, Simultaneous; AM, Apparent movement; H, High intensity; L, Low intensity; R, Right; L, Left.

Supplementary Information The online version contains supplementary material available at https://doi.org/10.3758/s13414-021-02278-1.

Acknowledgements We thank Alexander Billig, Raymond Van Ee, and John Rinzel for valuable feedback on earlier versions of this manuscript.

Author Contributions All authors were involved in the experimental design, discussion of the results and writing the manuscript. FD performed the experiments and analyzed the data.

Funding JR acknowledges support from an Engineering and Physical Sciences Research Council (EPSRC) New Investigator Award (EP/R03124X/1) and from the EPSRC Centre for Predictive Modelling in Healthcare (EP/N014391/1). 


\section{Declarations}

Conflict of interest The authors declare that they have no conflicts of interest.

Ethics approval Approval was obtained from the ethics committee of University of Exeter (eEMPS000058). The procedures used in this study adhere to the tenets of the Declaration of Helsinki.

Consent to participate Informed consent was obtained from all individual participants included in the study.

Consent for publication Not applicable.

Availability of data and materials The datasets generated and analyzed during the current study are available in the GitHub repository farzaneh-darki/Darki2021_perceptual: https:/github.com/ farzaneh-darki/Darki2021_perceptual.

Code availability Not applicable.

Open Practices Statement The data and materials for all experiments are available in the GitHub repository farzaneh-darki/ Darki2021_perceptual: https://github.com/farzaneh-darki/Darki2021_ perceptual. None of the experiments were preregistered.

Open Access This article is licensed under a Creative Commons Attribution 4.0 International License, which permits use, sharing, adaptation, distribution and reproduction in any medium or format, as long as you give appropriate credit to the original author(s) and the source, provide a link to the Creative Commons licence, and indicate if changes were made. The images or other third party material in this article are included in the article's Creative Commons licence, unless indicated otherwise in a credit line to the material. If material is not included in the article's Creative Commons licence and your intended use is not permitted by statutory regulation or exceeds the permitted use, you will need to obtain permission directly from the copyright holder. To view a copy of this licence, visit http://creativecommons. org/licenses/by/4.0/.

\section{References}

Barniv, D., \& Nelken, I. (2015). Auditory streaming as an online classification process with evidence accumulation. PloS one, 10(12).

Blake, R., Westendorf, D. H., \& Overton, R. (1980). What is suppressed during binocular rivalry?. Perception, 9(2), 223-231.

Blake, R. (1989). A neural theory of binocular rivalry. Psychological Review, 96(1), 145

Boring, E. G. (1942). Sensation and perception in the history of experimental psychology.

Brascamp, J. W., Van Ee, R., Pestman, W. R., \& Van Den Berg, A. V. (2005). Distributions of alternation rates in various forms of bistable perception. Journal of Vision, 5(4), 1-1.

Brascamp, J. W., Klink, P. C., \& Levelt, W.illem.J.M. (2015). The 'laws' of binocular rivalry: 50 years of Levelt's propositions. Vision Research, 109, 20-37.

Burtt, H. E. (1917). Tactual illusions of movement. Journal of Experimental Psychology, 2(5), 371.

Carter, O., Konkle, T., Wang, Q., Hayward, V., \& Moore, C. (2008). Tactile rivalry demonstrated with an ambiguous apparent-motion quartet. Current Biology, 18(14), 1050-1054.
Cao, R., Braun, J., \& Mattia, M. (2014). Stochastic accumulation by cortical columns may explain the scalar property of multistable perception. Physical Review Letters, 113(9), 098103.

Cao, R., Pastukhov, A., Aleshin, S., Mattia, M., \& Braun, J. (2020). Instability with a purpose: how the visual brain makes decisions in a volatile world. bioRxiv.

Cao, R., Pastukhov, A., Mattia, M., \& Braun, J. (2016). Collective activity of many bistable assemblies reproduces characteristic dynamics of multistable perception. Journal of Neuroscience, 36(26), 6957-6972.

Clarey, J. C., Tweedale, R., \& Calford, M. B. (1996). Interhemispheric modulation of somatosensory receptive fields: evidence for plasticity in primary somatosensory cortex. Cerebral Cortex, 6(2), 196-206.

Conrad, V., Vitello, M. P., \& Noppeney, U. (2012). Interactions between apparent motion rivalry in vision and touch. Psychological Science, 23(8), 940-948.

Denham, S. L., Farkas, D., Van Ee, R., Taranu, M., Kocsis, Z., Wimmer, M., ..., Winkler, I. (2018). Similar but separate systems underlie perceptual bistability in vision and audition. Scientific Reports, 8(1), 1-10.

Deutsch, D. (1974). An auditory illusion. Nature, 251(5473), 307-309.

Eshel, N., Ruff, C. C., Spitzer, B., Blankenburg, F., \& Driver, J. (2010). Effects of parietal TMS on somatosensory judgments challenge interhemispheric rivalry accounts. Neuropsychologia, 48(12), 3470-3481.

Faul, F., Erdfelder, E., Lang, A. G., \& Buchner, A. (n.d.) A flexible statistical power analysis program for the social, behavioral and biomedical sciences. Behavior Research Methods.

Ferrington, D. G., \& Rowe, M. A. R. K. (1980). Differential contributions to coding of cutaneous vibratory information by cortical somatosensory areas I and II. Journal of Neurophysiology, 43(2), 310-331.

Fox, R., \& Herrmann, J. (1967). Stochastic properties of binocular rivalry alternations. Perception \& Psychophysics, 2(9), 432436.

Gengerelli, J. A. (1948). Apparent movement in relation to homonymous and heteronymous stimulation of the cerebral hemispheres. Journal of Experimental Psychology, 38(5), 592.

Haladjian, H. H., Anstis, S., Wexler, M., \& Cavanagh, P. (2020). The tactile quartet: Comparing ambiguous apparent motion in tactile and visual stimuli. Perception, 49(1), 61-80.

Hämäläinen, H., Hiltunen, J., \& Titievskaja, I. (2002). Activation of somatosensory cortical areas varies with attentional state: an fMRI study. Behavioural Brain Research, 135(1-2), 159-165.

Hlushchuk, Y., \& Hari, R. (2006). Transient suppression of ipsilateral primary somatosensory cortex during tactile finger stimulation. Journal of Neuroscience, 26(21), 5819-5824.

Hupé, J.-M., \& Rubin, N. (2003). The dynamics of bi-stable alternation in ambiguous motion displays: a fresh look at plaids. Vision Research, 43(5), 531-548.

Iwamura, Y., Taoka, M., \& Iriki, A. (2001). Book review: Bilateral activity and callosal connections in the somatosensory cortex. The Neuroscientist, 7(5), 419-429.

Kastrup, A., Baudewig, J., Schnaudigel, S., Huonker, R., Becker, L., Sohns, J. M., ..., Witte, O. W. (2008). Behavioral correlates of negative bold signal changes in the primary somatosensory cortex. NeuroImage, 41(4), 1364-1371.

Kirman, J. H. (1974). Tactile apparent movement: The effects of interstimulus onset interval and stimulus duration. Perception \& Psychophysics, 15(1), 1-6.

Korvenoja, A., Wikstrom, H., Huttunen, J., Virtanan, J., Laine, P., Aronen, H. J., ..., Ilmoniemi, R. J. (1995). Activation of ipsilateral primary sensorimotor cortex by median nerve stimulation. Neuroreport, 6(18), 2589-2593. 
Lathrop, R. G. (1966). First-order response dependencies at a differential brightness threshold. Journal of Experimental Psychology, 72(1), 120.

Lederman, S. J., \& Klatzky, R. L. (2009). Haptic perception: A tutorial. Attention, Perception, \& Psychophysics, 71(7), 1439-1459.

Lederman, S. J., \& Jones, L. A. (2011). Tactile and haptic illusions. IEEE Transactions on Haptics, 4(4), 273-294.

Leopold, D. A., \& Logothetis, N. K. (1999). Multistable phenomena: changing views in perception. Trends in Cognitive Sciences, 3(7), 254-264.

Levelt, W. J.M. (1965). On binocular rivalry. Ph.D. Thesis Van Gorcum Assen.

Liaci, E., Bach, M., van Elst, L. T., Heinrich, S. P., \& Kornmeier, J. (2016). Ambiguity in tactile apparent motion perception. PloS One, 11(5).

Logothetis, N. K., Leopold, D. A., \& Sheinberg, D. L. (1996a). What is rivalling during binocular rivalry?. Nature, 380(6575), 621.

Logothetis, N. K., Leopold, D. A., \& Sheinberg, D. L. (1996b). What is rivalling during binocular rivalry?. Nature, 380(6575), 621.

Maldjian, J. A., Gottschalk, A., Patel, R. S., Detre, J. A., \& Alsop, D. C. (1999). The sensory somatotopic map of the human hand demonstrated at 4 tesla. NeuroImage, 10(1), 55-62.

Meng, M., \& Tong, F. (2004). Can attention selectively bias bistable perception? differences between binocular rivalry and ambiguous figures. Journal of Vision, 4(7), 2-2.

Meso, A. I., Rankin, J., Faugeras, O., Kornprobst, P., \& Masson, G. S. (2016). The relative contribution of noise and adaptation to competition during tri-stable motion perception. Journal of Vision, 16(15), 6-6.

Moreno-Bote, R., Shpiro, A., Rinzel, J., \& Rubin, N. (2010). Alternation rate in perceptual bistability is maximal at and symmetric around equi-dominance. Journal of Vision, 10(11), 1-1.

Nihashi, T., Naganawa, S., Sato, C., Kawai, H., Nakamura, T., Fukatsu, H., ..., Aoki, I. (2005). Contralateral and ipsilateral responses in primary somatosensory cortex following electrical median nerve stimulation-an fMRI study. Clinical Neurophysiology, 116(4), 842-848.

Noachtar, S., Lüders, H. O., Dinner, D. S., \& Klem, G. (1997). Ipsilateral median somatosensory evoked potentials recorded from human somatosensory cortex. Electroencephalography and Clinical Neurophysiology/Evoked Potentials Section, 104(3), 189198.

Pressnitzer, D., \& Hupé, J.-M. (2006). Temporal dynamics of auditory and visual bistability reveal common principles of perceptual organization. Current biology, 16(13), 1351-1357.

Ramachandran, V. S., \& Anstis, S. M. (1983). Perceptual organization in moving patterns. Nature, 304(5926), 529-531.

Rankin, J., Sussman, E., \& Rinzel, J. (2015). Neuromechanistic model of auditory bistability. PLoS Computational Biology, 11(11), e1004555.

Romo, R., \& Salinas, E. (2003). Flutter discrimination: neural codes, perception, memory and decision making. Nature Reviews Neuroscience, 4(3), 203-218.

Saal, H. P., Wang, X., \& Bensmaia, S. J. (2016). Importance of spike timing in touch: an analogy with hearing?. Current Opinion In Neurobiology, 40, 142-149.
Schnitzler, A., Salmelin, R., Salenius, S., Jousmäki, V., \& Hari, R. (1995). Tactile information from the human hand reaches the ipsilateral primary somatosensory cortex. Neuroscience Letters, 200(1), 25-28.

Sherrick, C. E., \& Rogers, R. (1966). Apparent haptic movement. Perception \& Psychophysics, 1(3), 175-180.

Sherrick, C. E. (1968). Bilateral apparent haptic movement. Perception \& Psychophysics, 4(3), 159-160.

Sterzer, P., Kleinschmidt, A., \& Rees, G. (2009). The neural bases of multistable perception. Trends in Cognitive Sciences, 13(7), 310 318.

Talbot, W. H., Darian-Smith, I., Kornhuber, H. H., \& Mountcastle, V. B. (1968). The sense of flutter-vibration: comparison of the human capacity with response patterns of mechanoreceptive afferents from the monkey hand. Journal of Neurophysiology, 31(2), 301-334.

Tommerdahl, M., Hester, K. D., Felix, E. R., Hollins, M., Favorov, O. V., Quibrera, P. M., \& Whitsel, B. L. (2005). Human vibrotactile frequency discriminative capacity after adaptation to $25-\mathrm{Hz}$ or $200-\mathrm{Hz}$ stimulation. Brain Research, 1057(1-2), 19.

Toppino, T. C. (2003). Reversible-figure perception: Mechanisms of intentional control. Perception \& Psychophysics, 65(8), 12851295.

Van Ee, R., Noest, A. J., Brascamp, J. W., \& van den Berg, A. V. (2006). Attentional control over either of the two competing percepts of ambiguous stimuli revealed by a two-parameter analysis: Means do not make the difference. Vision research, 46(19), 3129-3141.

van Ee, R. (2009). Stochastic variations in sensory awareness are driven by noisy neuronal adaptation: evidence from serial correlations in perceptual bistability. JOSA A, 26(12), 26122622.

Wilson, H. R. (2003a). Computational evidence for a rivalry hierarchy in vision. Proceedings of the National Academy of Sciences, 100(24), 14499-14503.

Wilson, H. R. (2003b). Computational evidence for a rivalry hierarchy in vision. Proceedings of the National Academy of Sciences, 100(24), 14499-14503.

Wolfe, J. M. (1996). Resolving perceptual ambiguity. Nature, 380(6575), 587-588.

Wuerger, S., Shapley, R., \& Rubin, N. (1996). "On the visually perceived direction of motion"? by Hans Wallach: 60 years later. Perception, 25(11), 1317-1367.

Yau, J. M., Olenczak, J. B., Dammann, J. F., \& Bensmaia, S. J. (2009). Temporal frequency channels are linked across audition and touch. Current Biology, 19(7), 561-566.

Zhou, Y. H., Gao, J. B., White, K. D., Merk, I., \& Yao, K. (2004). Perceptual dominance time distributions in multistable visual perception. Biological Cybernetics, 90(4), 256-263.

Zhou, W., \& Chen, D. (2009). Binaral rivalry between the nostrils and in the cortex. Current Biology, 19(18), 1561-1565.

Publisher's note Springer Nature remains neutral with regard to jurisdictional claims in published maps and institutional affiliations. 